\title{
Research Note \\ Supersolar metal abundances and the Broad Line Region of Narrow-line Seyfert 1 galaxies
}

\author{
S. Komossa ${ }^{1}$ and S. Mathur ${ }^{2}$ \\ 1 Max-Planck-Institut für extraterrestrische Physik, Postfach 1603, 85740 Garching, Germany \\ 2 The Ohio State University, 140 West 18th Avenue, Columbus, OH 43210, USA
}

Received 12 February 2001 / Accepted 5 June 2001

\begin{abstract}
Narrow-line Seyfert 1 galaxies (NLSy1) are intriguing due to their extreme continuum and emission line properties which are not yet well understood. This paper is motivated by the recent suggestion that NLSy1 galaxies might be young active galactic nuclei (AGNs) and that they may have supersolar metal abundances. We have examined the stability of broad emission line region (BLR) clouds under isobaric perturbation and its dependence on gas metal abundances. We show that supersolar metallicity increases the range where multiple phases in pressure balance may exist. This is particularly important for NLSy1s with steep X-ray spectra where it delays the trend of hindrance of a multi-phase equilibrium. Finally, the role of warm absorbers in the context of this scenario is assessed.
\end{abstract}

Key words. galaxies: active - galaxies: nuclei - galaxies: abundances - galaxies: Seyfert

\section{Introduction}

\subsection{Narrow-line Seyfert 1 galaxies}

$\mathrm{X}$-ray and optical observations of the last decade revealed a new sub-class of active galaxies, termed Narrow-line Seyfert 1 galaxies (NLSy1s hereafter). NLSy1 galaxies are intriguing due to their extreme continuum and emission line properties. Their optical broad lines are narrower than in "normal" Seyferts and they show strong FeII emission. Their X-ray spectra are often unusually soft. Many properties of AGN were shown to correlate strongly with each other. The strongest variance, often referred to as "eigenvector 1 " is defined by the correlation between the width of the $\mathrm{H} \beta$ emission line and the strength of the [OIII] emission line, and the anticorrelation with the ratio $\mathrm{FeII} / \mathrm{H} \beta$ (Boroson \& Green 1992). NLSy1 galaxies are placed at one extreme end of eigenvector 1 and, in general, their X-ray softness correlates with their optical properties. There is evidence that the X-ray properties of NLSy1 galaxies are best explained by a high accretion rate close to the Eddington rate (Pounds et al. 1995). Recently, it has been proposed that the reason for the high accretion rate is the age (Mathur 2000a). In this scenario NLSy1 galaxies with high accretion rate are AGNs in an early evolutionary phase. There are several lines of evidence which indicate

Send offprint requests to: S. Komossa,

e-mail: skomossa@xray.mpe.mpg.de that NLSy1 galaxies may have super-solar gas phase metal abundances (Mathur 2000a,b; see our Sect. 3 for details) and thus represent the analogue to high-redshift quasars which are believed to be young objects, and which show supersolar metallicity as well (e.g., Hamann \& Ferland 1993; Dietrich et al. 2000).

In this Note, we discuss the influence of supersolar gas phase metal abundances on the possible multi-phase equilibrium of the broad-line clouds in NLSy1 galaxies.

\subsection{Multi-phase cloud equilibrium: previous approaches}

Different mechanisms for the confinement/stability of the broad line clouds have been studied over the years. The model originally investigated by Krolik et al. (1981; KMT hereafter) was a two-phase BLR cloud model, consisting of cold line-emitting clouds $\left(T \simeq 10^{4} \mathrm{~K}\right)$ in pressure balance with a hot inter-cloud medium $\left(T \simeq 10^{8} \mathrm{~K}\right)$. The original KMT model faces problems when a more recent AGN continuum shape is used, in the sense that a pressure balance between a cold, photoionization heated, and a hot, Compton heated phase no longer exists. In addition, depending on the continuum shape, the hot phase may have lower temperature $\left(T \simeq 10^{7} \mathrm{~K}\right)$ and produce observable signatures due to its optical thickness (e.g., Elvis et al. 1985; Fabian et al. 1986; Rees 1987). 
However, more recent studies have established the presence of an additional stable region of intermediate temperature (Reynolds \& Fabian 1995; Komossa \& Fink 1997a,b), and the recent discovery of warm absorbers located in that intermediate region have revived the interest in multi-phase cloud models. It is also interesting to note that recent Chandra HETG observations of NGC 4151 confirmed the existence (Elvis et al. 1990) of a high-temperature X-ray emitting plasma, interpreted in terms of a hot intercloud medium (Ogle et al. 2000). This observation can be well explained by the classical KMT model, due to the very flat X-ray spectrum of NGC 4151 (Komossa 2001b).

With respect to Narrow-line Seyfert 1 galaxies, Brandt et al. (1994) suggested that the relatively narrow optical BLR lines of these galaxies might be traced back to the hindrance of BLR multi-phase equilibrium in the presence of steep X-ray spectra. Model calculations of Komossa \& Meerschweinchen (2000, KM2000 hereafter; see also Komossa \& Fink 1997a) confirmed the idea. However, recent observations suggest that other mechanisms might be at work (e.g., a small black hole mass would lead to narrow lines in NLSy1 galaxies). Motivated by the recent evidence for supersolar metal abundances in NLSy1 galaxies we extend here the earlier calculations to explore in more detail the influence of gas abundances. We show that supersolar metallicity increases the range where multiple phases in pressure balance may exist, and that it delays the trend of hindrance of a multi-phase equilibrium due to steep $\mathrm{X}$-ray spectra.

\section{Model calculations and results}

\subsection{The model}

We used the photoionization code Cloudy (Ferland 1993) to carry out the calculations. In order to obtain the cloud temperature as a function of pressure the following assumptions about the cloud properties were made:

Constant gas density $\left(\log n_{\mathrm{H}}=9.5\right)$ and solar element abundances according to Grevesse \& Anders (1989) were adopted, and then varied relative to the solar value. It was assumed that there is no strong temperature gradient across the clouds. The clouds were illuminated by the continuum of a central point-like energy source with a spectral energy distribution (SED) from the radio to the gamma-ray region as in Komossa \& Schulz (1997). This mean Seyfert $\mathrm{SED}^{1}$ consists of piecewise powerlaws with, in particular, an energy index $\alpha_{\mathrm{uv}-\mathrm{x}}=-1.4$ in the EUV. The X-ray photon index $\Gamma_{\mathrm{x}}$ was varied to cover the range typically observed in NLSy1 galaxies.

The cloud temperature after each model run was extracted, as a function of the ionization parameter. The

${ }^{1}$ Rodriguez-Pascual et al. (1997) collected IR - X-ray fluxes of Seyfert and NLSy1 galaxies and concluded that both are generally very similar concerning luminosities in different energy bands except that NLSy1s tend to be underluminous in the UV. ionization parameter is defined as

$U=\frac{Q}{4 \pi r^{2} n c}$

where $n$ is the gas density, $r$ the distance of the gas cloud from the continuum source, $c$ the speed of light, and $Q$ the number rate of photons above the Lyman limit $\left(\nu_{0}=10^{15.512} \mathrm{~Hz}\right)$.

The stability of broad line clouds to isobaric perturbations can be examined by studying the behavior of temperature $T$ as a function of pressure. If the temperature is multi-valued for constant pressure, and the gradient of the equilibrium curve is positive, several phases may exist in pressure balance. Results are shown in Fig. 1 where variations in $U / T$ correspond to variations in $1 /$ pressure. In the upper part of Fig. 1, we have plotted a sequence of curves which were derived by using different X-ray slopes in the SEDs which illuminate the clouds. The powerlaw index changes from $\Gamma_{\mathrm{x}}=-1.5$ to -4 from left to right, and the multi-valued nature of the curves disappears for steep $\mathrm{X}$-ray spectra. Due to the relatively weaker X-ray flux and the fact, that the value of $U$ is dominated by the EUV flux near the Lyman limit, the gas remains longer in the phase which is characterized by photoionization-heating and collisional-excitation-cooling. The same holds for a continuum with a hot soft X-ray excess (KM2000). For comparison with results obtained using the mean Seyfert SED, we have collected multi-wavelength continuum observations of the NLSy1 galaxy NGC 4051 (Komossa \& Fink 1997a and references therein), and plotted the corresponding equilibrium curve in Fig. 1 (see next section for details).

Metal abundances, which affect the cooling of the gas, were then varied. We find that supersolar metal abundances increase the range where a multi-phase equilibrium is possible, whereas subsolar abundances have the opposite effect (cf. the curve of Fig. 1 which refers to $\left.\Gamma_{\mathrm{x}}=-1.9\right)$. Whereas SEDs with an X-ray spectrum steeper than $\Gamma_{\mathrm{x}} \approx-2.5$ do no longer allow for multiple phases in pressure equilibrium if solar gas phase abundances are adopted, such equilibria are still possible in case of supersolar abundances.

In a next step, we selectively increased the neon and nitrogen abundances $\left(Z_{\mathrm{N}, \mathrm{Ne}}=3 \times Z_{\odot}\right)$, motivated by $\left(a_{1}\right)$ the observations of X-ray spectral features around $\sim 1.1-1.4 \mathrm{keV}$ which could partly be due to increased $\mathrm{Ne}$ absorption, $\left(a_{2}\right)$ optical evidence for overabundant $N$, and (b) the report of Fabian et al. (1986) that Ne has a particularly strong influence on the equilibrium curve (but see comment (ii) of our Sect. 3.3). We do not find any exceptional effects (Fig. 1), but note that modifications of the shape of the curve are dominated by Ne, not N.

\subsection{The BLR in NGC 4051}

We have constructed the equilibrium curves with the SED of the NLSy1 NGC 4051 as an input. The results for solar and super-solar abundances are shown in Fig. 1c. Below, we give some order-of-magnitude estimates of the parameters of the BLR of NGC 4051 in the context of our general 

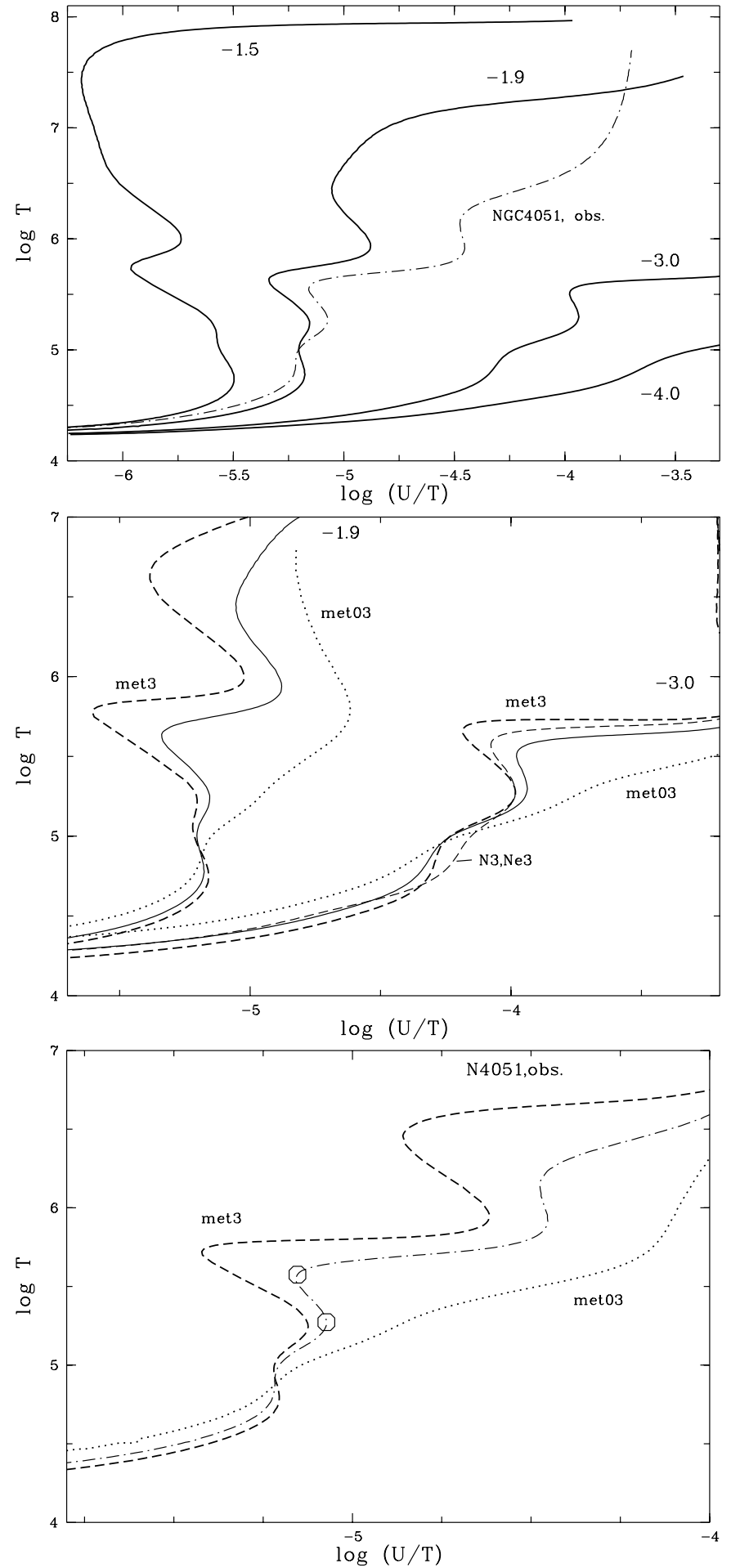

modeling approach. It is clear from the figure that a twophase equilibrium with common pressure does not exist for solar abundances. On the other hand, such an equilibrium is possible if abundances are super-solar. In the context of the BLR cloud model, the lower temperature region corresponds to the BLR clouds while the the confining medium is in the hot region. From Fig. 1c, we find that the ionization parameter of the BLR spans a range between $\log U=0.12$ and $\log U=0.30$. Reverberation mapping observations can determine the distance of the
Fig. 1. Equilibrium gas temperature $T$ against $U / T$ for various SEDs incident on the gas and metal abundances of the gas. Upper panel: solid black lines: mean Seyfert input continuum with $\Gamma_{\mathrm{x}}=-1.5,-1.9,-3.0$ and -4.0 as labeled in the graph; dot-dashed line: observed multi-wavelength continuum of the NLSy1 galaxy NGC 4051 with $\Gamma_{\mathrm{x}}=-2.3$. Solar gas phase abundances were adopted. Middle panel: sector of upper panel, showing the dependence on abundances. Solid lines: mean Seyfert input continuum with $\Gamma_{\mathrm{x}}=-1.9$ and -3.0 replotted for comparison; thick dashed lines: overabundant metals $\left(Z=3 \times Z_{\odot}\right)$, thin dashed line: selectively enhanced nitrogen and neon abundances of $3 \times$ solar, dotted lines $=$ underabundant metals $\left(Z=0.3 \times Z_{\odot}\right)$. Lower panel: results specific to NGC 4051. The dot-dashed line corresponds, again, to the observed multi-wavelength continuum of NGC 4051 using solar abundances. The open circles mark the location of NGC 4051's warm absorber, observed at two different epochs (Nov. 1993, Komossa \& Fink 1997a; and Nov. 1991, KM2000). The values were derived from one-component warm absorber fits to the ROSAT X-ray spectrum of this galaxy, assuming solar metallicity. Dashed and dotted line have the same meaning as above.

BLR from the nucleus accurately. In NGC 4051 it was found to be $1.6 \times 10^{16} \mathrm{~cm}$ (Peterson et al. 2000). Using Eq. (1), the density in the BLR can be constrained to be $8.7<\log n<9.1^{2}$. If such a model for a BLR is valid for all NLSy1s, then it implies that BLR densities in NLSy1s

${ }^{2}$ This density estimate is based on a value of $Q=1.6 \times 10^{52}$ $1 / \mathrm{s}$ of NGC 4051, obtained using a piecewise powerlaw as SED. "Photon counting arguments" based on the $\mathrm{H} \beta$ luminosity suggest that this is a lower limit on $Q$ (Komossa \& Fink 1997a). A higher value of $Q$ would increase the inferred density. 
are low and ionization parameters are higher than the "standard" value $(\log U \approx-2)$. Higher densities and lower $U$ may be obtained for even higher metallicities.

These results are consistent with the conclusions of Rodriguez-Pascual et al. (1997) who argue in favour of smaller density and higher ionization parameter in NLSy1 galaxies. Note, however, that they did not consider super-solar metallicities in their model.

The next question is whether the parameters of the warm absorber in NGC 4051 are consistent with being in pressure equilibrium with the BLR clouds. This is a non-trivial question in the case of NGC 4051 with highly variable ionizing continuum. Presenting non-equilibrium photoionization calculations, Nicastro et al. (1999) estimated that the warm absorber in NGC 4051 is at a distance of $\sim 1.3 \pm 0.3 \times 10^{16} \mathrm{~cm}$ from the nucleus, which is similar to that of the BLR. Therefore, pressure equilibrium with the BLR clouds is possible. A more detailed comparison will be possible, once X-ray observations of higher spectral resolution of NGC 4051 become available, in combination with photoionization calculations that include time-dependent effects.

\section{Discussion}

\subsection{Evidence for supersolar metallicity in NLSy1 galaxies}

Mathur (2000a; see also Kawaguchi \& Aoki 2000) suggested NLSy1s to be young/rejuvenated active galaxies. In analogy to high- $z$ quasars they may have super-solar metal abundances, as indicated by a number of observations:

An overabundance of iron was suggested to explain the strong optical FeII emission in NLSy1s (Collin \& Joly 2000). Supersolar Fe or Ne provide a possible explanation for the peculiar absorption features around $\sim 1 \mathrm{keV}$ seen in some NLSy1 galaxies (e.g., Ulrich et al. 1999; Turner et al. 1999). Overabundant Fe was also discussed to explain the strength of the FeK $\alpha$ lines (Fabian 1999). Nitrogen may be overabundant as well (e.g., Wills et al. 2000; Mathur 2000b).

\subsection{Properties of the BLR in NLSy1 galaxies}

X-ray spectra steeper than $\Gamma_{\mathrm{x}} \approx-2.5$ completely remove the multi-phase equilibrium (see Fig. 7 of KM2000), whereas a number of NLSy1s with even steeper soft X-ray spectra have been observed. So, within the context of the multi-phase model it is important to ask, which mechanism could alter that trend.

In addition, the study of Rodriguez-Pascual et al. (1997) shows that NLSy1s do have broad $U V$ lines $\left(F W H M \simeq 5000 \mathrm{~km} \mathrm{~s}^{-1}\right)$; only the optical BLR lines appear smaller $\left(F W H M \lesssim 2000 \mathrm{~km} \mathrm{~s}^{-1}\right)$. The existence of the broad UV lines has to be explained.

We find that supersolar metal abundances halt complete removal of multiple phases in pressure balance, and therefore allow for the presence of a BLR.

\subsection{Multi-phase BLR model}

Before we discuss consequences of the models considered here, we first give some cautious comments on the general modeling assumptions. (i) It was assumed that there is no strong temperature gradient across the clouds. For very large column densities that would no longer be true. (ii) The detailed shape of the equilibrium curve in the intermediate-temperature region, where metals are important, depends on the details of the heating-cooling processes and reflects to some extent the completeness with which these are implemented in the code used (see Ferland et al. 1998; Kingdon \& Ferland 1999). (iii) The EUV spectrum also has an important influence on the shape of the curves but it has already been studied previously (Fabian et al. 1986, KM2000).

Furthermore, it has to be kept in mind that a number of alternative BLR models have been studied. The BLR clouds might be confined by mechanisms other than thermal pressure (e.g. magnetic fields could play a role; Rees 1987), or they might not be confined at all. Confinement is unnecessary if the BLR clouds are continuously produced $^{3}$. These alternatives to cloud-confinement models face problems as well, though. For instance, BLR cloud counting arguments made the bloated stars and related scenarios unlikely (at least as explanation for the low-ionization component of the BLR; Dietrich et al. 1999).

With all this in mind, we come back to variants of the Krolik-McKee-Tarter (KMT) model. It is interesting to note that recent Chandra observations gave further input to the "classical" confinement scenarios, based on the detection of extended high-temperature X-ray emission in an AGN (Ogle et al. 2000), interpreted as hot intercloud medium.

The study of Rodriguez-Pascual et al. (1997) showed that NLSy1 galaxies possess broad emission lines in the UV. They explained the different line-widths measured in the optical and UV by a matter-bounded BLR, as discussed by Shields et al. (1995), in NLSy1 galaxies. This fits to the KMT model approach, which predicts a thin shell-like BLR.

\subsection{Warm absorbers in NLSy1 galaxies}

The region in which the temperature is multi-valued for constant pressure is preserved by supersolar metallicity in NLSy1s. If the X-ray spectra are not too steep, a pressure balance between a cold phase (the BLR in NLSy1s) and a hotter intermediate phase is possible (even if the very hot phase is absent).

This intermediate region has previously been associated with ionized absorbers (e.g., Reynolds \& Fabian 1995; Komossa \& Fink 1997a,b; Elvis 2000a,b; see Fig. 1 of Komossa 2001 for a summary).

\footnotetext{
${ }^{3}$ e.g., Murray \& Chiang (1995) (disk-driven winds; see also Dietrich et al. 1999), Edwards (1980), Alexander \& Netzer (1994) (bloated stars), Perry \& Dyson (1985) (cloudlet creation by radiative shocks).
} 
The suggestion that NLSy1s are young objects with a high accretion rate makes the presence of a large amount of (ionized) gas in the nucleus likely (Mathur 2000b). Indeed warm absorbers have been detected in quite a number of NLSy1 galaxies (e.g., Brandt et al. 1997; Komossa \& Fink 1997a; Hayashida 1997; Komossa \& Bade 1998; Iwasawa et al. 1998; Ulrich et al. 1999; Vaughan et al. 1999), or have been suggested to explain some unusual spectral/variability properties of NLSy1s (e.g., Komossa \& Meerschweinchen 2000). Some studies indicate that ionized absorbers are somewhat less abundant in NLSy1s than in Seyferts (Leighly 1999). The reason could be a higher degree of ionization of the absorbers which makes them less easily detectable in NLSy1s. The OVII absorbers, seen in some NLSy1 galaxies (e.g., IRAS $17020+4544)$, which might at first glance suggest a low degree of ionization, can easily be explained by the presence of a second warm absorber component which is located at larger distances from the nucleus, and which is mixed with dust (Komossa \& Bade 1998).

\section{Summary and conclusions}

We have presented a study of the influence of metallicity on the multi-phase equilibrium in photoionized gas. We find that supersolar metallicity increases the range over which a multi-phase medium in pressure balance may exist. In objects with steep X-ray spectra, like NLSy1s, such an equilibrium is not possible if gas metallicity is less than or equal to solar. As a result a pressure confined BLR cannot exist. This problem is alleviated if metallicity is supersolar. This offers further credence to the suggestion that NLSy1s may have supersolar metallicity. High resolution X-ray spectroscopy, together with optical/UV spectroscopy, is required to derive elemental abundances and to address these issues in more detail. The results of this Note are also of relevance to high-redshift quasars, since their BLR clouds show supersolar metal abundances.

Acknowledgements. This work is supported in part by the NASA grant NAG5-8913 (LTSA) to SM. We thank Gary Ferland for providing Cloudy. Preprints of this and related papers can be retrieved at http://www .xray.mpe.mpg.de/ skomossa/

\section{References}

Alexander, T., \& Netzer, H. 1994, MNRAS, 270, 781

Boroson, T. A., \& Green, R. F. 1992, ApJS, 80, 109

Brandt, W. N., Fabian, A. C., Nandra, K., Reynolds, C. S., \& Brinkmann, W. 1994, MNRAS, 271, 958

Brandt, W. N., Mathur, S., Reynolds, C., \& Elvis, M. 1997, MNRAS, 292, 497

Collin, S., \& Joly, M. 2000, New Astr. Rev., 44, 531

Dietrich, M., Wagner, S. J., Courvoisier, T. J.-L., Bock, H., \& North, P. 1999, A\&A, 351, 31

Dietrich, M., \& Wilhelm-Erkens, U. 2000, A\&A, 354, 17

Edwards, A. C. 1980, MNRAS, 198, 757

Elvis, M. 2000a, New Astr. Rev., 44, 559

Elvis, M. 2000b, ApJ, 545, 63
Elvis, M., Fassnacht, C., Wilson, A. S., \& Briel, U. 1990, ApJ, 361,459

Elvis, M., Wilkes, B., \& Tananbaum, H. 1985, ApJ, 292, 357

Fabian, A. C. 1999, in Proc: Observational and theoretical progress in the study of Narrow Line Seyfert 1 Galaxies, held in Bad Honnef, Germany

Fabian, A. C., Guilbert, P. W., Arnaud, K. A., Shafer, R. A., Tennant, A. F., \& Ward, M. J. 1986, MNRAS, 218, 457

Ferland, G. J. 1993, University of Kentucky, Physics Department, Internal Report

Ferland, G. J., Korista, K. T., Verner, D. A., et al. 1998, PASP, 110,761

Grevesse, N., \& Anders, E. 1989, in Cosmic Abundances of Matter, ed. C. J. Waddington, New York: American Institute of Physics, AIP 183, 1

Hayashida, K. 1997, in Proc: Emission Lines in Active Galaxies - New Methods and Techniques, ed. B. M. Peterson, F.-Z. Cheng, \& A. S. Wilson, ASP Conf. Ser., 113, 40

Hamann, F., \& Ferland, G. J. 1993, ApJ, 418, 11

Iwasawa, K., Brandt, W. N., \& Fabian, A. C. 1998, MNRAS, 293,251

Kawaguchi, T., \& Aoki, K. 2000, PASJ, submitted

Kingdon, J. B., \& Ferland, G. J. 1999, ApJ, 516, L107

Komossa, S. 2001, in Proc: IX. Marcel Grossmann Meeting on General Relativity, Gravitation and Relativistic Field Theories, ed. V. Gurzadyan, et al., in press [astro-ph/0101289]

Komossa, S. 2001b, A\&A, 371, 507

Komossa, S., \& Bade, N. 1998, A\&A, 331, L49

Komossa, S., \& Fink, H. 1997a, A\&A, 322, 719

Komossa, S., \& Fink, H. 1997b, A\&A, 327, 483

Komossa, S., \& Meerschweinchen, J. 2000, A\&A, 354, 411 (KM2000)

Komossa, S., \& Schulz, H. 1997, A\&A, 323, 31

Krolik, J. H., McKee, C. F., \& Tarter, C. B. 1981, ApJ, 249, 422

Leighly, K. M. 1999, ApJS, 125, 317

Mathur, S. 2000a, MNRAS, 314, L17

Mathur, S. 2000b, New. Astr. Rev., 44, 469

Murray, N., \& Chiang, J. 1995, ApJ, 454, L105

Nicastro, F., Fiore, F., Perola, G. C., \& Elvis, M. 1999, ApJ, 511,184

Ogle, P. M., Marshall, H. L., Lee, J. C., \& Canizares, C. R., ApJ, 545, L81

Perry, J. J., \& Dyson, J. E. 1985, MNRAS, 213, 665

Peterson, B. M., McHardy, I. M., Wilkes, B. J., et al. 2000, 542,161

Pounds, K. A., Done, C., \& Osborne, J. P. 1995, MNRAS, 277, L5

Rees, M. J. 1987, MNRAS, 228, 47

Reynolds, C. S., \& Fabian, A. C. 1995, MNRAS, 273, 1167

Rodriguez-Pascual, P. M., Mas-Hesse, J. M., \& Santos-Lleo, M. 1997, A\&A, 327, 72

Shields, J. C., Ferland, G. J., \& Peterson, B. M. 1995, ApJ, 441,507

Turner, T. J., George, I., \& Netzer, H. 1999, ApJ, 526, 52

Ulrich, M. H., Comastri, A., Komossa, S., \& Crane, P. 1999, A\&A, 350, 816

Vaughan, S., Reeves, J., Warwick, R., \& Edelson, R. 1999, MNRAS, 309, 113

Wills, B. J., Shang, Z., \& Yuan, J. M. 2000, New Astr. Rev., 44,511 